\title{
Structural Findings in the Basal Ganglia in Genetically Determined and Idiopathic Parkinson's Disease
}

\author{
Kathrin Reetz, MD ${ }^{1,2}$ Christian Gaser, $\mathrm{PhD},{ }^{3}$ Christine Klein, MD, ${ }^{1}$ Johannes Hagenah, MD, ${ }^{1}$ \\ Christian Büchel, MD, ${ }^{2,4}$ Stefan Gottschalk, MD, ${ }^{5}$ Peter P. Pramstaller, MD, ${ }^{6}$ \\ Hartwig R. Siebner, MD, ${ }^{2,7}$ and Ferdinand Binkofski, MD ${ }^{1,2 *}$ \\ ${ }^{1}$ Department of Neurology, University of Luebeck, Luebeck, Germany \\ ${ }^{2}$ Imaging Center NeuroImage Nord, Hamburg, Kiel, Lübeck, Germany \\ ${ }^{3}$ Department of Psychiatry, University of Jena, Jena, Germany \\ ${ }^{4}$ Department of Systems Neuroscience, University Medical Center Hamburg Eppendorf, Hamburg, Germany \\ ${ }^{5}$ Institute of Neuroradiology, University of Luebeck, Luebeck, Germany \\ ${ }^{6}$ Department of Neurology, Central Hospital and Genetic Medicine, EURAC-Research, Bolzano-Bozen, Italy \\ ${ }^{7}$ Department of Neurology, University of Kiel, Kiel, Germany
}

\begin{abstract}
A bilateral compensatory increase of basal ganglia (BG) gray matter value (GMV) was recently demonstrated in asymptomatic Parkin mutation carriers, who likely have an increased risk to develop Parkinson's disease (PD). We hypothesized BG morphological changes in symptomatic Parkin mutation carriers (sPARKIN-MC) and idiopathic PD patients (iPD) after the occurrence of PD symptoms, reflecting the breakdown of compensatory mechanisms. Nine sPAR$K I N-\mathrm{MC}, 14 \mathrm{iPD}$, and 24 controls were studied clinically and with voxel-based morphometry. Analysis of variance revealed mainly BG decrease of GMV in sPARKIN-MC and to a lesser extent in iPD. However, a slight increase in GMV was also found in the right globus pallidus externus in $\mathrm{S} P A R K I N-$ $\mathrm{MC}$ and in the right putamen in iPD. This may reflect a
\end{abstract}

structural correlate of functional compensation that can only partially be maintained when nigrostriatal neurodegeneration becomes manifest. Simple regression analyses with the UPDRS-III and disease duration score revealed a distinct more bilateral linear decrease of BG GMV in sPARKIN-MC than in iPD that may correspond to previous findings showing a symmetric reduction in putaminal ${ }^{18} \mathrm{~F}$-DOPA-uptake and bilateral manifestation of symptoms in sPARKIN-MC. In symptomatic PD, BG are subject to a progressive atrophy, which gradually increases with disease severity and duration. (c) 2008 Movement Disorder Society

Key words: basal ganglia; magnetic resonance imaging; Parkinson's disease; Parkin mutation carriers; voxel-based morphometry
Parkinson's disease (PD) is a common, slowly progressive neurodegenerative disorder primarily characterized by rigidity, tremor, and bradykinesia. Although the origin of PD currently remains unknown, in the majority of PD patients (idiopathic PD [iPD]), a total of about $2-3 \%$ of all cases can now be explained by a monogenic cause. Mutations in the Parkin gene are the

*Correspondence to: Ferdinand Binkofski, Department of Neurology, University of Luebeck, Ratzeburger Allee 160, 23538 Luebeck, Germany. E-mail: ferdinand.binkofski@neuro.uni-luebeck.de

Potential conflict of interest: None reported.

Received 7 December 2007; Revised 10 July 2008; Accepted 28 August 2008

Published online 29 September 2008 in Wiley InterScience (www. interscience.wiley.com). DOI: 10.1002/mds.22333 most common known single factor responsible for early-onset parkinsonism. ${ }^{1}$ This monogenic variant is often clinically ${ }^{2,3}$ and pathologically ${ }^{1,4}$ indistinguishable from iPD. Clinical observations, however, suggest that Parkin-associated PD tends to manifest earlier and more symmetric, have a milder course than iPD and better response to levodopa. ${ }^{3,5}$

In PD, motor symptoms are caused by a dysfunction of the cortico-basal-ganglia-loop due to progressive degeneration of nigrostriatal dopaminergic neurons. Correspondingly, positron emission tomography (PET) studies demonstrated in symptomatic Parkin mutation carriers (sPARKIN-MC), as well as in iPD patients, a reduction of the striatal 18 -fluorodopa $\left({ }^{18} \mathrm{~F}\right.$-DOPA) uptake indicating a presynaptic dopaminergic dysfunction. ${ }^{6-9}$ Notably, sPARKIN-MC showed a more sym- 
metric reduction of ${ }^{18} \mathrm{~F}$-DOPA uptake $\mathrm{e}^{7,8,10}$ and a slower progression in ${ }^{18} \mathrm{~F}$-DOPA PET than iPD patients. ${ }^{9}$

While ample PET data about PD patients are available, ${ }^{6,7,9}$ the results of MR-morphometric studies regarding the basal ganglia in iPD patients are still scarce and inconsistent: In iPD patient's normal ${ }^{11-14}$ or (partly) decreased $^{15-18}$ gray matter volume (GMV) of the basal ganglia were observed. In more detail, MRI-based studies on patients with advanced PD detected a decreased volume of the putamen ${ }^{15,16,18}$ and globus pallidus ${ }^{18}$ and in a voxel-based morphometry (VBM) study, an atrophy of the head of the left caudate was found. ${ }^{17}$

There are no morphometric MRI data on sPARKINMC available as yet. However, we recently demonstrated that asymptomatic Parkin mutation carriers (aPARKIN-MC) showed a bilateral increase of striatal GMV. ${ }^{19}$ In our aPARKIN-MC, who likely have an increased risk to develop $\mathrm{PD}$ and therefore may be regarded as a model for a presymptomatic stage of PD, PET revealed a latent presynaptic dopaminergic dysfunction. This led us propose that the "hypertrophy" in the basal ganglia may be a long-term consequence of compensatory mechanisms that successfully counteract the chronic dopaminergic striatal dysfunction. Alternatively, the morphometric changes may be a direct consequence of nigrostriatal dysfunction itself. ${ }^{19}$

Thus, the objective of the present study was to investigate the relationship between the severity and duration of motor symptoms in iPD and sPARKIN-MC with the extent and topography of basal ganglia GMV changes as revealed by VBM. Regarding our hypothesis, the initial "compensatory hypertrophy" in the basal ganglia should gradually taper off when PD has reached the symptomatic stage of the disease. More specifically, the more severely the patients are affected, the stronger the expected decrease in basal ganglia GMV.

\section{PATIENTS AND METHODS}

To address this issue, $9 \mathrm{~s} P A R K I N-\mathrm{MC}$ (two females, mean age: $52.3 \pm 3.8$ years) and $14 \mathrm{iPD}$ patients (eight females, mean age: $50.9 \pm 1.1$ years) were investigated, along with 24 age- and sex-matched controls (11 females, mean age: $52.1 \pm 1.5$ years). The sPAR$K I N-\mathrm{MC}$ and iPD patients were recruited from the outpatient movement disorders clinics at the Department of Neurology, University of Luebeck, in Germany and at the Department of Neurology, Central Hospital and Genetic Medicine, EURAC-Research, Bolzano-Bozen in Italy where the patients have been diagnosed and are followed up on a regular basis. All patients traveled to the study center in Luebeck and were person- ally interviewed and clinically examined using a standardized protocol. The motor section of the Unified Parkinson's Disease Rating Scale (UPDRS-III) was used to quantify extrapyramidal signs. Furthermore, all subjects underwent a standardized clinical psychiatric examination including the Mini-Mental State Examination (MMSE) for dementia. For practical reasons, all assessments and MRI scans were carried out in the onphase to minimize movement artifacts due to tremor. All volunteers had a normal neurological and neuropsychological examination as well as tested negative for mutations in the Parkin gene. All subjects and patients gave their informed written consent for participation in this study, which was approved by the local ethics committee.

$\mathrm{T}_{1}$-weighted FLASH-3D MR-images (echo time [TE] $=5$ milliseconds; repetition time $[\mathrm{TR}]=15$ milliseconds; flip angle $=30^{\circ}$; voxel size $1 \times 1 \times 1 \mathrm{~mm}^{3}$ ) were assessed on a $1.5 \mathrm{~T}$ scanner (Siemens, Symphony, Erlangen, Germany). Morphometric analysis was performed on a voxel-by-voxel basis using SPM2 software (FIL, London, UK, www.fil.ion.ucl.ac.uk/spm). Details of the VBM procedure are described elsewhere. ${ }^{19}$ Categorical comparisons were calculated between sPARKIN$\mathrm{MC}$, iPD patients, and their corresponding control group. Since we hypothesized that the severity and the duration of the disease is accompanied by reduced GMV, in a second step, simple regression analyses between morphometric data and clinical scores were performed using the SPM regression algorithms. Because our a priori hypothesis predicted structural changes in the striatum and the globus pallidus (GP), these regions were used as region-of-interest using the WFU-PickAtlas (ANSIR, Wake Forest University) ${ }^{20}$ as anatomical reference. Given our a priori hypothesis the statistical threshold of $P<0.005$ (uncorrected) was executed for the regions of interest. For the whole brain volume the statistical threshold was set at positive false discovery rate $(p \mathrm{FDR})<0.05$ after correction.

\section{RESULTS}

All sPARKIN-MC and iPD patients were clinically affected with Parkinsonism. There was no significant age difference between the sPARKIN-MC and iPD patients and their control groups $(P=0.80)$. There was no dementia in any group. The mean UPDRS-III score

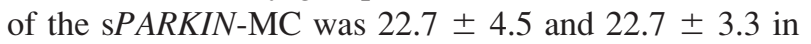
iPD patients. There was no significant difference between these two groups $(P=0.98)$ and there was also no significant difference regarding the affected side in the PD patients groups. Mean disease duration, defined 
TABLE 1. Characteristics of symptomatic Parkin mutation carriers and idiopathic Parkinson's disease patients

\begin{tabular}{|c|c|c|c|c|c|c|}
\hline No. & Mutations & Sex & Age (yr) & UPDRS-III & Affected side & Disease duration \\
\hline PARKIN 1 & $\operatorname{del} 1072+\operatorname{del} 1072$ & M & 54 & 33 & Both & 08 \\
\hline PARKIN 2 & $\operatorname{delEx} 7+\operatorname{delEx} 7$ & $\mathrm{~F}$ & 41 & 05 & Right & 06 \\
\hline PARKIN 3 & delEx7 + del1072 & M & 75 & 31 & Both & 11 \\
\hline PARKIN 4 & delEx7 + del1072 & M & 72 & 38 & Right & 23 \\
\hline PARKIN 5 & delEx2 & M & 44 & 13 & Left & 07 \\
\hline PARKIN 6 & $211 \mathrm{C}>\mathrm{T}$ & $\mathrm{F}$ & 41 & 45 & Left & 08 \\
\hline PARKIN 7 & delEx2-5 & M & 48 & 13 & Left & 10 \\
\hline PARKIN 8 & delEx4 +924C $>\mathrm{T}$ & M & 52 & 18 & Both & 37 \\
\hline PARKIN 9 & $\operatorname{delEx} 3-4+\operatorname{delEx} 7+\operatorname{delEx} 9$ & M & 44 & 08 & Right & 13 \\
\hline IPD 1 & None & $\mathrm{F}$ & 61 & 06 & n.a. & 04 \\
\hline IPD 2 & None & $\mathrm{F}$ & 57 & 04 & Right & 10 \\
\hline IPD 3 & None & $\mathrm{F}$ & 50 & 49 & Left & 14 \\
\hline IPD 4 & None & $\mathrm{F}$ & 54 & 30 & Right & 13 \\
\hline IPD 5 & None & $\mathrm{F}$ & 47 & 25 & Left & 16 \\
\hline IPD 6 & None & M & 52 & 32 & Right & 19 \\
\hline IPD 7 & None & M & 51 & 09 & Right & 10 \\
\hline IPD 8 & None & M & 49 & 27 & Left & 14 \\
\hline IPD 9 & None & M & 49 & 33 & Right & 14 \\
\hline IPD 10 & None & $\mathrm{F}$ & 50 & 10 & Right & 09 \\
\hline IPD 11 & None & $\mathrm{F}$ & 52 & 17 & Left & 12 \\
\hline IPD 12 & None & M & 49 & 22 & Right & 11 \\
\hline IPD 13 & None & $\mathrm{F}$ & 44 & 25 & Right & 09 \\
\hline IPD 14 & None & M & 48 & 30 & Left & 14 \\
\hline
\end{tabular}

\section{A) Categorical comparison}

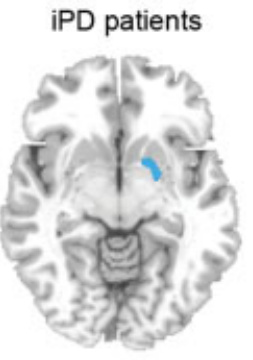

$z=-8$

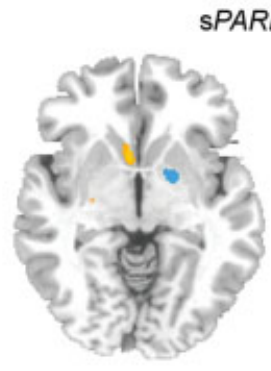

$z=-8$ SPARKIN-MC

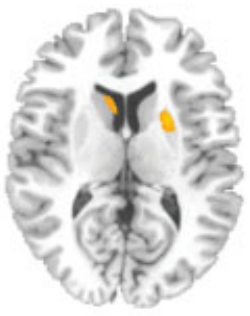

$z=12$

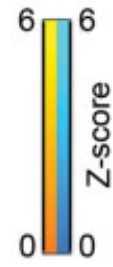

orange $=$ patients $<$ controls blue $=$ patients $>$ controls
B) Regression analyses with UPDRS-III

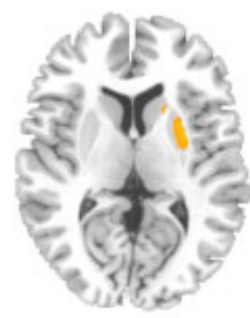

$z=13$

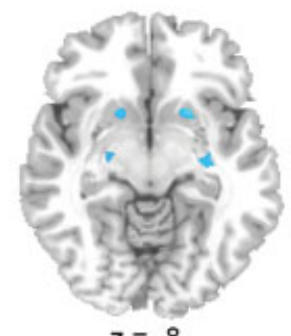

$z=-8$

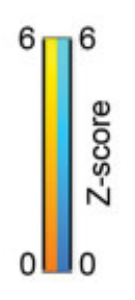

C) Regression analyses with disease duration

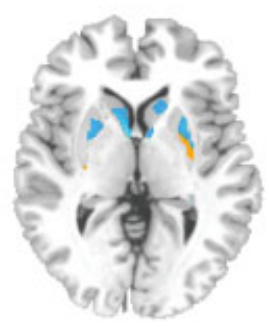

$z=3$

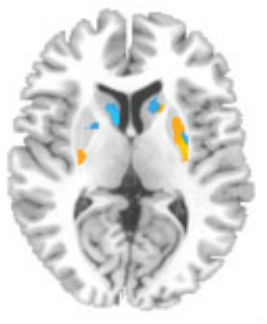

$z=9$

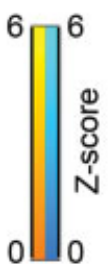

orange $=$ iPD

blue = $\mathrm{s} P A R K I N-M C$

FIG. 1. Structural changes in the basal ganglia in $S P A R K I N-M C$ and iPD patients. A: The categorical comparison revealed a decrease (orange) of GMV in the left caudate in $\mathrm{SPARKIN-MC}$ and a mild increase (blue) in the right globus pallidus externus in sPARKIN-MC and in the right putamen iPD patients. B: Simple regression analysis with the UPDRS-III score in SPARKIN-MC (orange) displayed a mild GMV decrease in the putamen bilaterally and in the right caudate. In the iPD group (blue) the GMV decrease was most prominent in the right putamen. C: In sPARKIN$\mathrm{MC}$ (orange) the GMV were decreased in the caudate and putamen bilaterally in the simple regression analysis with the disease duration, whereas in the iPD group (blue) the greatest GMV decrease was found in the right putamen again; the left putamen was however slightly affected. 
as time from symptom onset, was $13.7 \pm 3.2$ years in sPARKIN-MC and $12.1 \pm 1.0$ years in iPD patients. There was also no significant difference between these two groups of patients $(P=0.59)$ (Table 1).

Analysis of variance (Fig. 1A, Table 2) revealed a slight increase in GMV in the right globus pallidus externus (GPe) adjacent to the right putamen in $\mathrm{s} P A R$ KIN-MC relative to their controls. The relative GMV increase was smaller in symptomatic patients compared with the increases that we had previously found in asymptomatic PARKIN-MC. ${ }^{19}$ However, the more predominant finding was a reduction in GMV in the head of the left caudate nucleus and right putamen in $\mathrm{s} P A R$ $K I N$-MC and to a lesser extent also in the left caudate in iPD patients. These findings were verified in the following regression analyses.

Simple regression analysis (Fig. 1B,C, Table 2) was used to test for linear changes in GMV with UPDRSIII score or disease duration. In SPARKIN-MC, the GMV in both the putamina and the right caudate decreased significantly with the UPDRS-III score. In addition, the head of the caudate nucleus and the putamen showed a bilateral decrease in GMV with disease duration. In iPD patients predominantly the right putamen showed a linear decrease in GMV with the UPDRS-III score and with disease duration.

\section{DISCUSSION}

Using VBM, that is capable of discovering subtle, regionally specific changes in GMV, we found two major differences in the pathoanatomic patterns in the basal ganglia in genetically determined and iPD patients. First, the results confirmed our hypothesis that at the symptomatic stage in both genetically determined and idiopathic PD, the basal ganglia are subject to a progressive atrophy, which gradually increases with severity and duration of symptoms in PD. However, in the context of our recent results in asymptomatic heterozygous PARKIN-MC, ${ }^{19}$ the possibly residual, slight increase of putaminal and GPe GMV in both groups of PD patients may indicate remaining compensatory mechanisms. Second, although both groups of patients showed basal ganglia alterations in GMV in the categorical comparisons and relative decreases in GMV associated with the UPDRS-III score or disease duration as revealed by the regression analyses, the regional expression of disease-related atrophy was not similar. These findings show structural differences between symptomatic PARKIN-MC and patients with iPD that may reflect clinical and metabolic findings in these groups as identified in previous studies.
TABLE 2. Coordinates and gray matter values of symptomatic Parkin mutation carriers and iPD patients

\begin{tabular}{|c|c|c|c|c|c|}
\hline \multirow[b]{2}{*}{ Region } & \multirow[b]{2}{*}{ Side } & \multicolumn{3}{|c|}{$\begin{array}{l}\text { MNI coordinates } \\
\text { in } \mathrm{mm}\end{array}$} & \multirow[b]{2}{*}{$Z$-score } \\
\hline & & $x$ & $y$ & $z$ & \\
\hline \multicolumn{6}{|c|}{$\begin{array}{l}\text { Categorical comparison } \\
\text { sParkin- } M C<\text { controls }\end{array}$} \\
\hline Putamen & $\mathrm{R}$ & 23 & 7 & 12 & 3.13 \\
\hline Caudate & $\mathrm{L}$ & -7 & 9 & -2 & 3.22 \\
\hline \multicolumn{6}{|c|}{ sParkin-MC $>$ controls } \\
\hline $\begin{array}{l}\mathrm{GPe} \\
i P D<\text { contr }\end{array}$ & $\mathrm{R}$ & 17 & 1 & -7 & 2.59 \\
\hline $\begin{array}{l}\text { Caudate } \\
i P D>\text { contr }\end{array}$ & $\mathrm{L}$ & -17 & 13 & 18 & 2.62 \\
\hline GPe & $\mathrm{R}$ & 20 & 3 & -8 & 2.59 \\
\hline \multicolumn{6}{|c|}{$\begin{array}{l}\text { Regression analysis with UPDRS-III } \\
\text { sParkin-MC }\end{array}$} \\
\hline Putamen & $\mathrm{R}$ & 20 & 13 & -9 & 3.22 \\
\hline Putamen & $\mathrm{L}$ & -27 & 7 & -5 & 2.46 \\
\hline Caudate & $\mathrm{R}$ & 16 & -16 & 22 & 2.44 \\
\hline \multicolumn{6}{|l|}{$i P D$} \\
\hline Putamen & $\mathrm{R}$ & 27 & 3 & 13 & 3.07 \\
\hline \multicolumn{6}{|c|}{$\begin{array}{l}\text { Regression analysis with disease duration } \\
\text { sParkin-MC }\end{array}$} \\
\hline Putamen & $\mathrm{R}$ & 30 & 15 & 1 & 3.83 \\
\hline Putamen & $\mathrm{L}$ & -31 & -10 & -6 & 2.49 \\
\hline Caudate & $\mathrm{R}$ & 15 & 9 & 16 & 3.78 \\
\hline Caudate & $\mathrm{L}$ & -16 & -5 & 23 & 4.71 \\
\hline \multicolumn{6}{|l|}{$i P D$} \\
\hline Putamen & $\mathrm{R}$ & 29 & -9 & 9 & 3.05 \\
\hline
\end{tabular}

iPD, idiopathic Parkinson's disease patients; GPe, globus pallidus externus.

In more detail, the clinical regression analyses between the individual values of the UPDRS-III and disease duration and the morphometric data revealed a strong negative correlation with the basal ganglia. Previous MR-morphometric studies in iPD patients showed inconsistent results: The GMV of the basal ganglia was shown to be either normal ${ }^{11-14}$ or (partly) decreased $^{15,16,18,21}$ in iPD patients compared to controls. These discrepant structural findings may be due to different usage of methodology (MRI-based volumes or region of interest analysis [VOI/ROI], VBM) and differences in the severity and duration of the disease of the investigated PD population.

The observation that the severity and the duration of the disease are accompanied by striatal GMV changes may indicate a dynamically developing process in the striatum. In the context of our hypothesis of compensation for the basal ganglia dysfunction, the atrophy may now reflect parts of a "decompensation process," resulting in the loss of basal ganglia GMV ${ }^{15-18}$ and the appearance of clinical symptoms. Although the presence of a basal ganglia atrophy in PD patients is still a matter of controversy, ${ }^{11-14}$ it is conceivable given the 
increasing intensity of the nigro-striatal degeneration in PD. ${ }^{19}$ This is in accordance with postmortem studies showing a loss of striatal neurons in PD patients. ${ }^{22,23}$

The more symmetrically distributed pattern of degeneration in $\mathrm{S} P A R K I N-\mathrm{MC}$ corresponds to previous PET findings showing a symmetric reduction in putaminal ${ }^{18}$ F-DOPA-uptake ${ }^{7,8,10}$ and preferentially bilateral manifestation of symptoms in sPARKIN-MC. ${ }^{1,3}$ The differential pattern in SPARKIN-MC may be attributed to the slower progression of PD symptoms and a longer presymptomatic course of the disease, which may produce different degenerative and adaptive changes in the basal ganglia tissue.

As already suggested for the presymptomatic period in aPARKIN-MC, ${ }^{19}$ we assume that the partially increased basal ganglia GMV in iPD and sPARKIN-MC indicates a possible active compensatory mechanism for the chronic dopaminergic deficit resulting in basal ganglia dysfunction. ${ }^{7,9}$ Therefore, we propose that presynaptic dopaminergic basal ganglia dysfunction in sPARKIN$\mathrm{MC}$ and iPD patients leads initially to a chronic increase in neuronal activity within the basal ganglia and an increase in GMV of the hyperactive structures. This increase in GMV might represent a long-term consequence of adaptive plasticity in the striatum that allows for a compensation of the dopaminergic deficit in the striatal motor circuit still present after the occurrence of symptoms. Although the exact nature of the structural changes in the basal ganglia remains to be elucidated, our morphometric findings suggest a dynamic development of structural changes during the course of PD.

Acknowledgments: The study was supported by grants of the Deutsche Forschungsgemeinschaft (RE 2841/1-1 [KR], Kl1134-2-2 and K1-1134-3-1 [travel expenses]), the Parkinson's Disease Foundation (genetical investigations, clinical and genetical investigation of subjects), and the South Tyrolean Parkinson Association (recruitment of subjects, clinical assessment of subjects). CB (01GO0510), HS (01GO0511), and FB (01GO05102) were supported by a structural grant of the Bundesministerium für Bildung und Forschung (BMBF) to NeuroImageNord. CK, HS, and FB were further sponsored by the EU-FP6-Grant (GENEPARK; EU-LSHB-CT-2006-037544). CK is supported by a Lichtenberg Grant from the Volkswagen Foundation. KR (E06-2008) and CK are supported by intramural grants from the Medical Faculty, University of Luebeck.

\section{REFERENCES}

1. Lucking CB, Durr A, Bonifati V, et al. Association between early onset Parkinson's disease and mutations in the parkin gene. French Parkinson's Disease Genetics Study Group. N Engl J Med 2000;342:1560-1567.

2. Klein C, Pramstaller PP, Kis B, et al. Parkin deletions in a family with adult onset, tremor-dominant parkinsonism: expanding the phenotype. Ann Neurol 2000;48:65-71.
3. Lohmann E, Periquet M, Bonifati V, et al. How much phenotypic variation can be attributed to parkin genotype? Ann Neurol 2003;54:176-185.

4. Farrer M, Chan P, Chen R, et al. Lewy bodies and parkinsonism in families with parkin mutations. Ann Neurol 2001;50:293-300.

5. Kann M, Jacobs H, Mohrmann K, et al. Role of parkin mutations in 111 community-based patients with early-onset parkinsonism. Ann Neurol 2002;51:621-625.

6. Broussolle E, Lucking CB, Ginovart N, Pollak P, Remy P, Durr A. [18 F]-dopa PET study in patients with juvenile-onset PD and parkin gene mutations. Neurology 2000;55:877-879.

7. Hilker R, Klein C, Ghaemi M, et al. Positron emission tomographic analysis of the nigrostriatal dopaminergic system in familial parkinsonism associated with mutations in the parkin gene. Ann Neurol 2001;49:367-376.

8. Hilker R, Klein C, Hedrich $\mathrm{K}$, et al. The striatal dopaminergic deficit is dependent on the number of mutant alleles in a family with mutations in the parkin gene: evidence for enzymatic parkin function in humans. Neurosci Lett 2002;323:50-54.

9. Khan NL, Brooks DJ, Pavese N, et al. Progression of nigrostriatal dysfunction in a parkin kindred: an [18F]dopa PET and clinical study. Brain 2002;125(Part 10):2248-2256.

10. Khan NL, Valente EM, Bentivoglio AR, et al. Clinical and subclinical dopaminergic dysfunction in PARK6-linked parkinsonism: an 18F-dopa PET study. Ann Neurol 2002;52:849-853.

11. Ghaemi M, Hilker R, Rudolf J, Sobesky J, Heiss WD. Differentiating multiple system atrophy from Parkinson's disease: contribution of striatal and midbrain MRI volumetry and multi-tracer PET imaging. J Neurol Neurosurg Psychiatry 2002;73:517-523.

12. Schulz JB, Skalej M, Wedekind D, et al. Magnetic resonance imaging-based volumetry differentiates idiopathic Parkinson's syndrome from multiple system atrophy and progressive supranuclear palsy. Ann Neurol 1999;45:65-74.

13. Burton EJ, McKeith IG, Burn DJ, Williams ED, O'Brien JT. Cerebral atrophy in Parkinson's disease with and without dementia: a comparison with Alzheimer's disease, dementia with Lewy bodies and controls. Brain 2004;127(Part 4):791-800.

14. Huber SJ, Shuttleworth EC, Christy JA, Chakeres DW, Curtin A, Paulson GW. Magnetic resonance imaging in dementia of Parkinson's disease. J Neurol Neurosurg Psychiatry 1989;52:12211227.

15. Alegret M, Junque C, Pueyo R, et al. MRI atrophy parameters related to cognitive and motor impairment in Parkinson's disease. Neurologia 2001;16:63-69.

16. Krabbe K, Karlsborg M, Hansen A, et al. Increased intracranial volume in Parkinson's disease. J Neurol Sci 2005;239:45-52.

17. Brenneis C, Seppi K, Schocke MF, et al. Voxel-based morphometry detects cortical atrophy in the Parkinson variant of multiple system atrophy. Mov Disord 2003;18:1132-1138.

18. O'Neill J, Schuff N, Marks WJ, Jr, Feiwell R, Aminoff MJ, Weiner MW. Quantitative 1H magnetic resonance spectroscopy and MRI of Parkinson's disease. Mov Disord 2002;17:917927.

19. Binkofski F, Reetz K, Gaser C, et al. Morphometric fingerprint of asymptomatic Parkin and PINK1 mutation carriers in the basal ganglia. Neurology 2007;69:842-850.

20. Maldjian JA, Laurienti PJ, Kraft RA, Burdette JH. An automated method for neuroanatomic and cytoarchitectonic atlasbased interrogation of fMRI data sets. Neuroimage 2003;19: 1233-1239.

21. Brenneis C, Bosch SM, Schocke M, Wenning GK, Poewe W. Atrophy pattern in SCA2 determined by voxel-based morphometry. Neuroreport 2003;14:1799-1802.

22. Braak H, Ghebremedhin E, Rub U, Bratzke H, Del Tredici K. Stages in the development of Parkinson's disease-related pathology. Cell Tissue Res 2004;318:121-134.

23. Bugiani O, Perdelli F, Salvarani S, Leonardi A, Mancardi GL. Loss of striatal neurons in Parkinson's disease: a cytometric study. Eur Neurol 1980;19:339-344. 